\title{
Green Synthesis and In vitro Antioxidant Activity of 6-aryl-Quinazolin-4(3H)-ones via Suzuki Cross Coupling
}

\section{SHANKARAIAH PAGILLA ${ }^{1 *}$, SIDDHARTHA MARUPATI ${ }^{1}$ and KISHORE NANI ${ }^{2}$}

\author{
'Department of Freshman Engineering, Vardhaman College of Engineering, Shamshabad, \\ Kacharam, Hyderabad, Telangana-500018, India. \\ ${ }^{2}$ Department of Science and Humanities, MLR Institute of Technology, Dundigal, \\ Hyderabad, Telangana-500043, India. \\ ${ }^{*}$ Corresponding author E-mail: shankarpagilla@gmail.com
}

http://dx.doi.org/10.13005/ojc/330645

(Received: October 25, 2017; Accepted: November 15, 2017)

\begin{abstract}
Suzuki cross coupling reaction based synthetic method for the preparation of 6-arylquinazolin-4(3H)-ones (3a-h) in ethylene glycol dimethyl ether is described by coupling of 6-iodo2-phenyl-3-methyl-quinazolin-4(3H)-one (1) with various 6-aryl boronic acids 3 under microwave irradiation in between These compounds were screened for anti-oxidant activity.
\end{abstract}

Keywords: 6-aryl-Quinazolin-4(3H)-one, aryl boronic acid, $\mathrm{Pd}\left(\mathrm{PPh}_{3}\right)_{4}, 1,1$-diphenyl-2picrylhydrazil(DPPH), ABTS* + (2, 2'-azino-bis (3-ethylben - zthiazoline- 6-sulphonic acid), Suzuki coupling, Ethylene glycol dimethyl ether, Microwave irradiation, Antioxidant activity.

\section{INTRODUCTION}

The recent literature is enriched with progressive findings about quinazolin- $4(3 H)$-one and its derivatives which exhibit a broad range of biological properties such as antioxidant ${ }^{1}$, anti-tumour ${ }^{2}$, anti-inflammatory ${ }^{3}$, anti-malarial ${ }^{4}$, anti-bacterial ${ }^{5}$ and anticonvulsant activity ${ }^{6}$. Further, quinazolin-4(3H)-one have also been investigated scaffold for the synthesis of various drugs and their intermediates ${ }^{7}$.

Cross coupling reaction is an significant method of generating carbon-carbon bonds in organic compounds, which is catalyzed by various transition metals. In the past three decades, carboncarbon bond construction reaction has allowed chemists to produce complex molecular structures of various interests including total synthesis of natural products ${ }^{8}$, medicinal chemistry and industrial process development ${ }^{9}$.

In modern days, Microwave-Assisted Organic Synthesis (MAOS) has fascinated the interest of synthetic chemists. The rate of a reaction is speed up under microwave irradiation compared to conventional heating.

Insight of quinazolin-4(3H)-one moiety and cross coupling reaction, we have in the previous reported the successful use of Kumada crosscoupling reactions for the synthesis of 6 -aryl-quinazolin-4 $(3 H)$-ones derivatives ${ }^{10}$. However, this coupling is related with extended 
reaction times and with low yields. In search of our previous investigations toward developing more proficient protocol for the synthesis of 6-aryl-quinazolin-4(3H)-one derivatives, we optimized the Suzuki reaction conditions under microwave irradiation and conventional heating. It was envisaged that the microwave irradiation would enhance the rate of reaction, thereby reducing time.

Here in, we report the coupling of 6-iodo3-methyl-2-phenyl-quinazolin-4(3H)-one (1) with aryl boronic acids in presence of $\mathrm{Pd}\left(\mathrm{PPh}_{3}\right)_{4}$ as catalyst derivatives under microwave irradiation as well as conventional heating conditions and evaluated their in vitro antioxidant property.

\section{Experimental section}

IR spectra for all the compounds were recorded in solid $\mathrm{KBr}$ on infra cold model 337 PerkinElmer instrument. Melting points were measured in open capillary tubes and are uncorrected. The ${ }^{1} \mathrm{H}$ NMR (400 MHz) and ${ }^{13} \mathrm{C} \mathrm{NMR} \mathrm{(75} \mathrm{MHz)} \mathrm{spectra}$ were recorded on Varian Gemini 400 or Bruker 75 in $\mathrm{CDCl}_{3}$ using tetramethylsilane as internal standard. The purity of all obtained compounds was checked by thin-layer chromatography. High resolution Mass spectra were recorded on Q-TOF mass spectrometer. Multisynth series microwave system (Milestone) were used for Suzuki reaction.

\section{General procedure for the preparation of 3a-h via Suzuki cross coupling reaction Microwave heating}

The 6- iodoquinazolinone $1(0.5 \mathrm{mmol})$ and boronic acid $(0.55 \mathrm{mmol})$ were dissolved ethylene glycol dimethyl ether in a microwave vial. $\mathrm{Pd}\left(\mathrm{PPh}_{3}\right)_{4}$ (0.02 mmol) and $\mathrm{Na}_{2} \mathrm{CO}_{3}(1.25 \mathrm{mmol})$ were added, and the reaction mixture was irradiated in a microwave apparatus at $80^{\circ} \mathrm{C}, 250 \mathrm{~W}$ for 20 minute. After the reaction mixture was cooled to ambient temperature, the product was filtered, the filtrate was concentrated, and the crude mixture was purified by silica gel column chromatography using hexane/ ethyl acetate (88/12) as eluent.

\section{Conventional heating}

The 6-iodoquinazolinone $1(0.5 \mathrm{mmol})$, boronic acid $2(0.55 \mathrm{mmol})$ and $\mathrm{Na}_{2} \mathrm{CO}_{3}(1.25 \mathrm{mmol})$ were dissolved in ethylene glycol dimethyl ether in a seal tube. After degassing reaction mixture,
$\mathrm{Pd}\left(\mathrm{PPh}_{3}\right)_{4}(0.02 \mathrm{mmol})$ and were added. And the reaction mixture was heated at $80^{\circ} \mathrm{C}, 250 \mathrm{~W}$ for overnight. After the reaction mixture was cooled to ambient temperature, the product was filtered, the filtrate was concentrated, and the crude mixture was purified by silica gel column chromatography using hexane/ethyl acetate (88/12) as eluent.

\section{Assay of anti-oxidant property Lipid peroxidation assay}

In vitro lipid peroxidation of the compounds was determined by the described method $^{11,12}$. Briefly, $1 \mathrm{~mL}$ of rat liver microsomal fraction was added to $1.0 \mathrm{~mL}$ of $150 \mathrm{mM}$ Tris- $\mathrm{HCl}$ buffer ( $\mathrm{pH}$ 7.4) containing various concentrations (50, 100 and $150 \mu \mathrm{g} / \mathrm{mL}$ ) of test compounds, 0.2 $\mathrm{mL} \mathrm{FeCl}_{3}(1 \mathrm{mM})$ and $0.2 \mathrm{~mL}$ ascorbic acid (0.5 $\mathrm{mM}$ ) to induce lipid peroxidation. The mixtures were incubated at $37^{\circ} \mathrm{C}$ for 30 minutes. At the end of the incubation, $0.5 \mathrm{ml}$ of glacial acetic acid and $0.5 \mathrm{ml}$ of $0.33 \%$ TBA were added to each mixture. The mixtures were kept in a water bath at $97^{\circ} \mathrm{C}$ for 45 min. at cooling, the pink chromogen was extracted with $2 \mathrm{~mL}$ of butanol. The absorbance of the organic layer was measured at $535 \mathrm{~nm}$, and the thiobarbituric acid reactive substances produced were estimated using the MDA standard curve. BHA was used as a control substance $(20 \mu \mathrm{g} / \mathrm{ml})$.

\section{DPPH (1, 1-Diphenyl-2-picrylhydrazyl) radical Scavenging activity}

The free radical scavenging activity of isolated compounds was measured by 1,1-diphenyl2-picryl- hydrazyl (DPPH) method described by Blois 1958. $0.1 \mathrm{mM}$ solution of DPPH in methanol was prepared and $1 \mathrm{ml}$ of this solution was added to $3 \mathrm{~mL}$ of various concentrations of $(50,100$ and $150 \mu \mathrm{g} / \mathrm{ml})$ test compounds and the reference compound $(20 \mu \mathrm{g} / \mathrm{ml})$. After $30 \mathrm{~min}$., absorbance was measured at $517 \mathrm{~nm}$. BHA was used as the reference material. All the tests were performed in triplicate. The percentage of inhibition was calculated by comparing the absorbance values of the control and test samples.

$\%$ DPPH radical scavenging $=[($ Absorbance of control - Absorbance of test sample)/ (Absorbance of control)] $\times 100$ 
ABTS $^{*}+\left(2,2^{\prime}\right.$-azino-bis (3-ethylben - zthiazoline6 -sulphonic acid) radical cation decolourisation assay

This method was carried out by well described method $^{13}$. ABTS ${ }^{*}+(54.8 \mathrm{mg})$ was dissolved in $50 \mathrm{~mL}$ of distilled water to $2 \mathrm{mM}$ concentration and potassium persulphate $(17 \mathrm{mM}, 0.3 \mathrm{~mL})$ was added. The reaction mixture was left to stand at room temperature overnight in dark before use. To $0.2 \mathrm{~mL}$ of various concentrations $(50,100$ and $150 \mu \mathrm{g} / \mathrm{mL}$ ) of test samples and reference standard $\mathrm{BHA}$ $(20 \mu \mathrm{g} / \mathrm{mL}) 1.0 \mathrm{~mL}$ of distilled DMSO and $0.16 \mathrm{~mL}$ of ABTS $^{*}+$ solution was added to make a final volume of $1.36 \mathrm{~mL}$. Absorbance was measured spectro-photometrically, after $20 \mathrm{~min}$. at $734 \mathrm{~nm}^{14}$.

\section{Spectral data}

3-Methyl-2,6-diphenylquinazolin-4(3H)one (3a): ${ }^{1} \mathrm{H}$ NMR $\left(400 \mathrm{MHz}, \mathrm{CDCl}_{3}\right): \delta 8.56(\mathrm{~s}, 1 \mathrm{H})$, $8.02(\mathrm{~d}, 1 \mathrm{H}, J=8.6 \mathrm{~Hz}), 7.82(\mathrm{~d}, 1 \mathrm{H}, J=8.6 \mathrm{~Hz})$, $7.72(\mathrm{~d}, 2 \mathrm{H}, J=7.5 \mathrm{~Hz}), 7.47-7.60(\mathrm{~m}, 7 \mathrm{H}), 7.38(\mathrm{~m}$, $1 \mathrm{H}), 3.53(\mathrm{~s}, 3 \mathrm{H}) ;{ }^{13} \mathrm{C} \mathrm{NMR}\left(75 \mathrm{MHz}, \mathrm{CDCl}_{3}\right): \delta 162.7$, $155.9,146.4,139.8,139.6,135.3,133.1,130.0$, 128.9, 128.8, 127.9, 127.8, 127.1, 124.5, 120.7, 34.1; IR (KBr): $1680 \mathrm{~cm}^{-1}$; ESI-HRMS: $[\mathrm{M}+\mathrm{H}]^{+} \mathrm{m} / \mathrm{z}$ calculated for $\mathrm{C}_{21} \mathrm{H}_{17} \mathrm{~N}_{2} \mathrm{O}$ : 313.1263; found at $\mathrm{m} / \mathrm{z}$ 313.1343. m.p. $105-106^{\circ} \mathrm{C}$.

3-Methyl-2-phenyl-6-(o-tolyl)quinazolin4(3H)-one (3b) : ${ }^{1} \mathrm{H}$ NMR $\left(400 \mathrm{MHz}, \mathrm{CDCl}_{3}\right): \delta 8.31$ (s, $1 \mathrm{H}), 7.73-7.80(\mathrm{~m}, 2 \mathrm{H}), 7.55-7.60(\mathrm{~m}, 5 \mathrm{H}), 7.31$ (m, 4H), $3.52(\mathrm{~s}, 3 \mathrm{H}), 2.32(\mathrm{~s}, 3 \mathrm{H}) ;{ }^{13} \mathrm{C}$ NMR $(75 \mathrm{MHz}$, $\left.\mathrm{CDCl}_{3}\right): \delta 162.8,156.1,146.1,140.9,140.5,135.6$, $135.4,135.3,130.0,128.9,128.0,127.8,127.2$, 126.8, 125.9, 120.3, 34.3, 20.5; IR (KBr): 1674 $\mathrm{cm}^{-1}$; ESI-HRMS: $[\mathrm{M}+\mathrm{H}]^{+} \mathrm{m} / \mathrm{z}$ calculated for $\mathrm{C}_{22} \mathrm{H}_{19}$ $\mathrm{N}_{2} \mathrm{O}$ : 327.1419; found at $\mathrm{m} / \mathrm{z} 327.1481$. m.p. $136-138^{\circ} \mathrm{C}$.

6-(4-Methoxyphenyl)-3-methyl-2phenylquinazolin-4(3H)-one (3c): ${ }^{1} \mathrm{H}$ NMR $\left(400 \mathrm{MHz}, \mathrm{CDCl}_{3}\right): \delta 8.51(\mathrm{~s}, 1 \mathrm{H}), 7.99(\mathrm{~m}, 2 \mathrm{H}), 7.57-$ $7.65(\mathrm{~m}, 7 \mathrm{H}), 7.02(\mathrm{~d}, 2 \mathrm{H}, J=7.9 \mathrm{~Hz}), 3.87(\mathrm{~s}, 3 \mathrm{H})$, $3.54(\mathrm{~s}, 3 \mathrm{H}) ;{ }^{13} \mathrm{C}$ NMR $\left(75 \mathrm{MHz}, \mathrm{CDCl}_{3}\right): \delta 162.7$, $159.5,155.7,145.8,139.4,135.2132 .8,132.0$, $130.0,128.8,128.2,128.0,127.8,123.7,120.6$, 114.3, 55.3, 34.2; IR (KBr): $1674 \mathrm{~cm}^{-1}$. ESI-HRMS: $[\mathrm{M}+\mathrm{H}]^{+} \mathrm{m} / \mathrm{z}$ calculated for $\mathrm{C}_{22} \mathrm{H}_{18} \mathrm{~N}_{2} \mathrm{O}_{2}: 343.13683$; found at $m / z$ 343.1448. m.p. $161-162^{\circ} \mathrm{C}$.
6-(2-Chlorophenyl)-3-methyl-2-phenylquinazolin4(3H)-one (3d): ${ }^{1} \mathrm{H}$ NMR $\left(400 \mathrm{MHz}, \mathrm{CDCl}_{3}\right): \delta 8.40$ (s, 1H), 8.05 (d, $1 \mathrm{H}, J=7.9 \mathrm{~Hz}), 7.91(\mathrm{~d}, 1 \mathrm{H}, J=7.9$ $\mathrm{Hz}), \quad 7.58-7.27(\mathrm{~m}, 9 \mathrm{H}), 3.55(\mathrm{~s}, 3 \mathrm{H}) .{ }^{13} \mathrm{C}$ NMR $\left(75 \mathrm{MHz}, \mathrm{CDCl}_{3}\right): \delta 162.5,156.4,146.5,139.2,138.3$, 135.7, 135.2, 132.5, 131.4, 130.17, 130.08, 129.07, 128.0, 127.34, 127.02, 127.4, 120.3, 34.3. ESIHRMS: $[\mathrm{M}+\mathrm{H}]^{+} \mathrm{m} / \mathrm{z}$ calculated for $\mathrm{C}_{21} \mathrm{H}_{16} \mathrm{ClN}_{2} \mathrm{O}$ : 347.0873; found at $m / z 347.0959$. m.p. $110-111^{\circ} \mathrm{C}$.

6-(2,3-Dichlorophenyl)-3-methyl-2phenylquinazolin-4(3H)-one (3e): ${ }^{1} \mathrm{H}$ NMR $\left(400 \mathrm{MHz}, \mathrm{CDCl}_{3}\right): \delta 8.50(\mathrm{~d}, 1 \mathrm{H}, J=2.1 \mathrm{~Hz}), 7.95$ (dd, $1 \mathrm{H}, J=8.5 \mathrm{~Hz}), 7.82(\mathrm{~d}, 1 \mathrm{H}, J=8.3 \mathrm{~Hz}), 7.55$ $7.60(\mathrm{~m}, 7 \mathrm{H}), 7.39(\mathrm{~m}, 1 \mathrm{H}), 3.54(\mathrm{~s}, 3 \mathrm{H}) ;{ }^{13} \mathrm{C}$ NMR $\left(75 \mathrm{MHz}, \mathrm{CDCl}_{3}\right): \delta 162.5,156.6,147.2,136.9,135.6$, $135.5,135.1,132.8,130.2,128.9,128.4,127.9$, 127.6, 125.6, 124.8, 34.4; IR (KBr): $1674 \mathrm{~cm}^{-1}$. ESIHRMS: $[\mathrm{M}+\mathrm{H}]^{+} \mathrm{m} / \mathrm{z}$ calculated for $\mathrm{C}_{21} \mathrm{H}_{15} \mathrm{Cl}_{2} \mathrm{~N}_{2} \mathrm{O}_{2}$ : 381.0483; found at $\mathrm{m} / \mathrm{z}$ 381.0549. m.p. $222-224^{\circ} \mathrm{C}$

6-(4-Fluorophenyl)-3-methyl-2phenylquinazolin-4(3H)-one (3f): ${ }^{1} \mathrm{H}$ NMR $\left(400 \mathrm{MHz}, \mathrm{CDCl}_{3}\right) \delta 8.49(\mathrm{~s}, 1 \mathrm{H}), 7.95(\mathrm{~d}, 1 \mathrm{H}, J=$ $8.4 \mathrm{~Hz}), 7.81(\mathrm{~d}, 1 \mathrm{H}, J=8.3 \mathrm{~Hz}), 7.55-7.69(\mathrm{~m}, 7 \mathrm{H})$, 7.15-7.199 (m, 2H), $3.51(\mathrm{~s}, 3 \mathrm{H}) ;{ }^{13} \mathrm{C}$ NMR $(75 \mathrm{MHz}$, $\left.\mathrm{CDCl}_{3}\right): \delta 162.6,156.4,146.4,138.7,135.7,135.2$, $132.9,130.0,128.8,127.9,124.2,120.6,115.9$, 115.6, 34.2; IR (KBr): $1680 \mathrm{~cm}^{-1}$. ESI-HRMS: $[\mathrm{M}+\mathrm{H}]^{+}$ $\mathrm{m} / z$ calculated for $\mathrm{C}_{21} \mathrm{H}_{16} \mathrm{CIN}_{2} \mathrm{O}: 347.0951$; found at $\mathrm{m} / \mathrm{z}$ 347.0959. m.p. $195-196^{\circ} \mathrm{C}$.

6-(3-Fluoro-4-methoxyphenyl)-3-methyl2-phenylquinazolin-4(3H)-one (3g): ${ }^{1} \mathrm{HNMR}$ $\left(400 \mathrm{MHz}, \mathrm{CDCl}_{3}\right) \delta 8.49 \mathrm{~s}(\mathrm{~s}, 1 \mathrm{H}), 7.95(\mathrm{~m}, 2 \mathrm{H})$ 7.44-7.69 $(\mathrm{m}, 7 \mathrm{H}), 7.07(\mathrm{~m}, 1 \mathrm{H}), 3.96(\mathrm{~s}, 3 \mathrm{H}), 3.55$ $(\mathrm{s}, 3 \mathrm{H}) ;{ }^{13} \mathrm{C} \mathrm{NMR}\left(75 \mathrm{MHz}, \mathrm{CDCl}_{3}\right): \delta 162.7,155.9$, 154.2, 150.9, 146.3, 138.2, 135.2, 132.6, 130.0, 128.8, 127.9, 123.9, 122.8, 120.6, 114.9, 114.6, 113.6, 56.3, 34.3; IR (KBr): $1679 \mathrm{~cm}^{-1}$; ESI-HRMS: $[\mathrm{M}+\mathrm{H}]^{+} \mathrm{m} / \mathrm{z}$ calculated for $\mathrm{C}_{21} \mathrm{H}_{15} \mathrm{FN}_{2} \mathrm{O}: 331.1168$; found at $m / z 331.1242$. m.p. $159-160{ }^{\circ} \mathrm{C}$.

3-Methyl-6-(naphthalen-1-yl)-2phenylquinazolin-4(3H)-one (3h): ${ }^{1} \mathrm{H}$ NMR $\left(400 \mathrm{MHz}, \mathrm{CDCl}_{3}\right) \delta 8.49(\mathrm{~d}, 1 \mathrm{H}, J=1.7 \mathrm{~Hz}), 7.86$ $7.95(\mathrm{~m}, 5 \mathrm{H}), 7.43-7.63(\mathrm{~m}, 9 \mathrm{H}), 3.54(\mathrm{~s}, 3 \mathrm{H}) ;{ }^{13} \mathrm{CNMR}$ $\left(75 \mathrm{MHz}, \mathrm{CDCl}_{3}\right) \delta 162.7,146.7,146.5,139.7,138.7$, $136.3,133.8,131.4,130.1,128.9,128.4,128.2$, 128.0, 127.6, 127.4, 126.4, 125.5, 125.4, 120.5, 34.3; 
IR (KBr): $1674 \mathrm{~cm}^{-1}$; ESI-HRMS: $[\mathrm{M}+\mathrm{H}]^{+} \mathrm{m} / \mathrm{z}$ calculated for $\mathrm{C}_{25} \mathrm{H}_{18} \mathrm{~N}_{2} \mathrm{O}$ : 363.1419; found at $\mathrm{m} / \mathrm{z}$ 363.1485. m.p. $111-113^{\circ} \mathrm{C}$.

\section{RESULT AND DISCUSSION}

6-Iodo-3-methyl-2-phenyl-quinazolin$4(3 H)$-one (1) was chosen as the scaffold which inturn was prepared in previous reported procedure. The compound 1 was characterized by ${ }^{1} \mathrm{H}$ NMR and HRMS spectral techniques ${ }^{10}$.

Microwave-assisted Suzuki Cross-coupling reaction of 6-iodo-quinazolin-4-(3H)-one (1) is explored for the first time with a set of eight aryl boronic acid 2 to provide 6-aryl-quinazolin-4-(3H)ones $3 a$. Primarily, the evaluation of solvent was studied using 6-iodo-quinazolin-4(3H)-one 1 (1.0 equiv) boronic acid $6 \mathrm{a}$ ( 1.1 equiv) and $\mathrm{Na}_{2} \mathrm{CO}_{3}$ 7 (1.1equiv). Employing solvents (Entry 1, 2, 3, 4, 5, and 6 ) resulted in the required compound $3 a$ with inferior yields. The reaction of $\mathbf{1}$ with aryl boronic acids $2 \mathrm{a}$ in presence of $10 \mathrm{~mol} \%$ of $\mathrm{Pd}\left(\mathrm{PPh}_{3}\right)_{4}$ as catalyst for $20 \mathrm{~min}$. in ethylene glycol dimethyl ether gave $3 a$ with $95 \%$ yield (scheme-1). The same product 6 a was also obtained (90\%) under conventional heating $\left(80^{\circ} \mathrm{C}\right)$ in toluene for 10 hours.

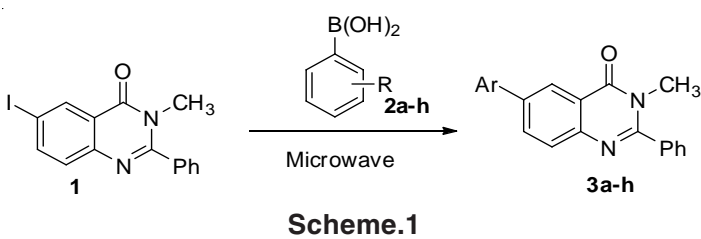

(Table 1, entry 1 ).

The spectral identification of $3 a$ has been discussed below. The IR $(\mathrm{KBr})$ spectrum of $3 a$ showed a strong absorption band at $1680 \mathrm{~cm}^{-1}$ corresponding to carbonyl group. The ${ }^{1} \mathrm{H}$ NMR spectrum $\left(\mathrm{CDCl}_{3}, 400 \mathrm{MHz}\right)$ of $3 a$ showed a singlet at $\delta 8.56$ for $\mathrm{C}-5$ proton, a doublet at $\delta 8.02$ corresponds to C-7 proton. A singlet peak at $\delta 3.53$ integrating for three protons is due to $\mathrm{N}-\mathrm{CH}_{3}$ group. The peaks for the remaining eleven aromatic protons observed at $\delta 7.82(\mathrm{~d}, J=8.6 \mathrm{~Hz}, 1 \mathrm{H}), \delta 7.72$ $(\mathrm{d}, J=7.50 \mathrm{~Hz}, 2 \mathrm{H}), \delta 7.47-7.60(\mathrm{~m}, 7 \mathrm{H})$ and in the range $\delta 7.38-7.41(\mathrm{~m}, 1 \mathrm{H}) .{ }^{13} \mathrm{C}-\mathrm{NMR}$ spectrum $\left(\mathrm{CDCl}_{3}, 100 \mathrm{MHz}\right)$ of $3 \mathrm{a}$ showed signals at $\delta 162.7$ due to carbonyl carbon, $\delta 155.9$ for $\mathrm{C}-2$ carbon, and a peak at $\delta 34.3$ due to $\mathrm{N}$-methyl carbon. Further
${ }^{13} \mathrm{C}$ NMR spectrum showed signals at $\delta 146.4$, $139.8,139.6,135.3,133.1,130.0,128.9,128.8$, $127.9,127.8,127.1,124.5,120.7$ due to the remaining aromatic carbons. The structure was further confirmed by high resolution Mass spectrum of $3 a$ indicates $[\mathrm{M}+\mathrm{H}]^{+}$peak at $\mathrm{m} / \mathrm{z} 313.1349$ due to the molecular formulae $\mathrm{C}_{21} \mathrm{H}_{17} \mathrm{~N}_{2} \mathrm{O}$.

This Suzuki coupling reaction of 1 was extended to remaining substituted phenyl boronic acids $2 \mathrm{~b}-\mathrm{h}$ and the corresponding 6 -arylquinazolin$4(3 \mathrm{H})$-ones $(3 \mathrm{~b}-\mathrm{h})$ were isolated in $93-95 \%$ yield (Scheme-1, Table-2). These compounds $3 \mathbf{b}-\mathbf{h}$ were characterized based on their IR, ${ }^{1} \mathrm{H}$ NMR, ${ }^{13} \mathrm{C}$ NMR and HRMS spectral data

Table.1: Optimized conditions for 3a compound with different solvents

\begin{tabular}{llll}
\hline \multirow{2}{*}{ Entry } & Solvent & \multicolumn{2}{c}{ Yield(\%) } \\
\cline { 3 - 4 } & & $80^{\circ} \mathrm{C}$ & $\mathrm{MW}$ \\
\hline 1. & DMF & 20 & 40 \\
2. & DMF:Water (4:1) & 40 & 60 \\
3. & Toulene & 37 & 40 \\
4. & Toluene:Water & 60 & 66 \\
5. & 1,4-Dioxane & 33 & 43 \\
6. & 1,4-Dioxane:Water & 62 & 71 \\
7. & Ethylene glycol & 85 & 95 \\
& dimethyl ether & & \\
\hline
\end{tabular}

\section{Assay of antioxidant property}

Lipid peroxidation assay

6-(2,3-Dichlorophenyl)-3-methyl-2-phenylquinazolin-4(3H)-one (3e) and 6-(3-Fluoro-4 methoxyphenyl)-3-methyl-2-phenylquinazolin$4(3 H)$-one $(3 \mathrm{~g})$ exhibited concentration-dependent $\mathrm{FeSO}_{4}$ induced lipid peroxidation and showed highest percentage of inhibition with that from other compounds and comparable to reference drug BHA. $\mathrm{P}<0.5$ (Table 4).

\section{DPPH radical scavenging activity}

Among the tested compounds 6-(2,3Dichlorophenyl)-3-methyl-2-phenylquinazolin$4(3 H)$-one (3e) and 6-(3-Fluoro-4-methoxyphenyl)3-methyl-2-phenylquinazolin-4(3H)-one $(3 \mathrm{~g})$ are showed highest percentage of inhibition which was comparable with standard BHA. 3-Methyl-2,6diphenylquinazolin-4(3H)-one (3a), 3-Methyl-2phenyl-6-(o-tolyl)quinazolin-4(3H)-one (3b), 6-(4- 
Table.2: Synthesis of 6-substituted quinazolinone using Suzuki cross coupling

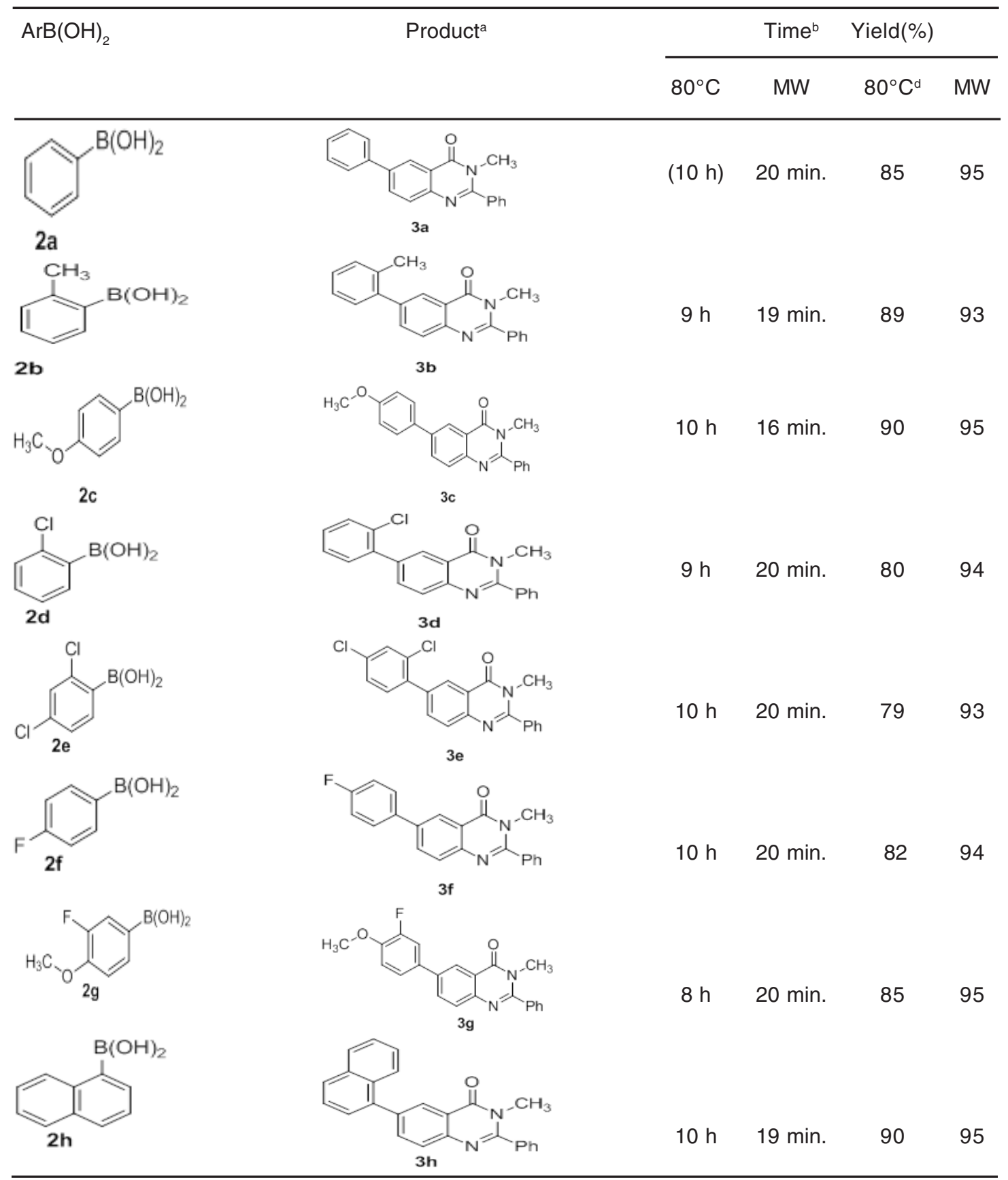

a. The products were characterized by $1 \mathrm{H}$ NMR, mass and IR spectra

b. Time for conventional heating $\left(80^{\circ} \mathrm{C}\right)$ reaction in mixture of toluene and water.

c. Isolated yields.

d. Yield after conventional heating $\left(80^{\circ} \mathrm{C}\right)$ reaction in mixture of toluene and water 


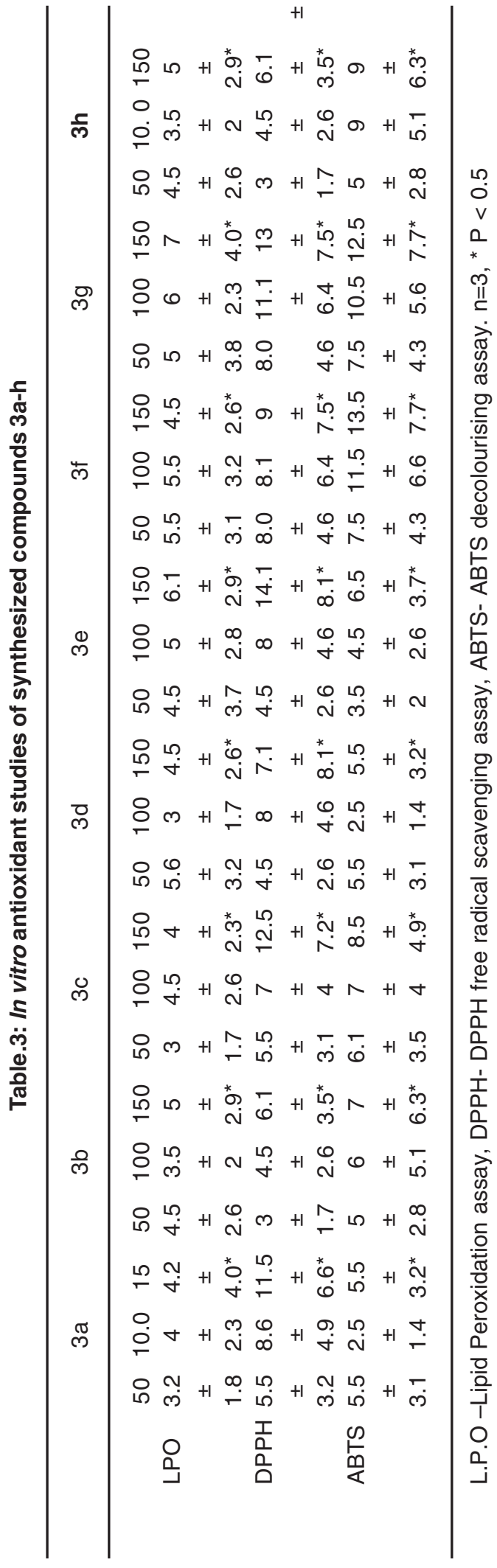

Methoxyphenyl)-3-methyl-2-phenylquinazolin4(3H)-one (3c), 6-(2-Chlorophenyl)-3-methyl-2phenylquinazolin-4(3H)-one (3d), 6-(4 Fluorophenyl)-3-methyl-2-phenylquinazolin-4(3H)-one (3f) and 3-Methyl-6-(naphthalen-1-yl)-2-phenylquinazolin-4(3H)-one (3h) are also possessed considerable anti-oxidant properties $\mathrm{P}<0.5$ (Table-3 ).

\section{$\mathrm{ABTS}^{*}+$ radical cation decolourisation assay}

Synthesized compounds showed good scavenging of $\mathrm{ABTS}^{*}+$ radical at all tested concentrations (Table-3). The highest inhibition was achieved with 6-(4-Fluorophenyl)-3-methyl-2phenylquinazolin-4(3H)-one (3f) comparing to other compounds $\mathrm{P}<0.5$. Whereas, 6 -(3-Fluoro-4methoxyphenyl)-3-methyl-2-phenylquinazolin$4(3 H)$-one $(3 \mathrm{~g})$ was the next compound showed significant at $150 \mu \mathrm{g} / \mathrm{mL}$ (Table-3).

\section{CONCLUSION}

A more efficient and novel C-C bond forming, Pd catalyzed Suzuki coupling reaction is developed as a new method for the preparation 6-aryl-quinazolin-4(3H)-ones (3a-h) under microwave irradiation conditions in ethylene glycol dimethyl ether has been achieved with good yields over conventional method. According to data obtained from the present study, these quinazolin-4(3H)-one derivatives could be considered as useful templates for further development to obtain In vitro antioxidant activity

\section{ACKNOWLEDGMENTS}

P. Shankaraiah and Siddhartha Marupati thank Vardhaman College, Shamshabad, Kacharam, Hyderabad, Telangana for providing financial assistance. 


\section{REFERENCES}

1. Priya, M. G. R.; Girija, K.; Ravichandran, N. Rasayan Journal of Chemistry, 2011,4, 418-424.

2. Noolvi, M. N.; Patel, H. M. Arabian Journal of Chemistry, 2013, 6, 35-48.

3. Salgýn-Gökpen, U.; Gökhan-Kelekçi, N.; Göktap, Ö.; Köysal, Y.; Kýlýç, E.; Ipýk, P.; Aktay, G.; Özalp, M.Bioorganic \& medicinal chemistry, 2007, 15, 5738-5751.

4. Birhan, Y. S.; Bekhit, A. A.; Hymete, A. BMC research notes, 2015, 8, 589.

5. Fathalla, O. A. E.-F. M.; Kassem, E. M.; Ibrahem, N. M.; Kamel, M. M. Acta Pol Pharm., 2008, 65, 11-20.

6. Paneersalvam, P.; Raj, T.; Ishar, M.; Singh, B.; Sharma, V.; Rather, B. Indian journal of pharmaceutical sciences, 2010, 72, 375.

7. Abida, P. N.; Arpanarana, M. International Journal of Pharmaceutical \& Biological Archive, 2011, 2, 1651-1657.

8. Chemler, S. R.; Trauner, D.; Danishefsky, S.
J. Angewandte Chemie International Edition 2001, 40, 4544-4568.

9. Johansson Seechurn, C. C.; Kitching, M. O.; Colacot, T. J.; Snieckus, V. Angewandte Chemie International Edition. 2012, 51, 5062-5085.

10. Shankaraiah, P.; Veeresham, S.; Bhavani, A. Russian Journal of General Chemistry, 2016, 86, 368-375.

11. Newman, D. J.; Cragg, G. M.; Snader, K. M. Natural product reports 2000, 17, 215-234.

12. Butler, M. S. Journal of natural products 2004, 67, 2141-2153.

13. Re, R.; Pellegrini, N.; Proteggente, A.; Pannala, A.; Yang, M.; Rice-Evans, C. Free radical biology and medicine 1999, 26, 1231-1237.

14. Magaldi, S.; Mata-Essayag, S.; De Capriles, C. H.; Perez, C.; Colella, M.; Olaizola, C.; Ontiveros, Y. International Journal of Infectious Diseases 2004, 8, 39-45. 